\title{
Erratum to: A comparison of heterosexual and LGBTQ cancer survivors' outlooks on relationships, family building, possible infertility, and patient-doctor fertility risk communication
}

\author{
Andrea M. Russell ${ }^{1}$ - Kathleen M. Galvin ${ }^{2}$ - Maya M. Harper ${ }^{3}$ - Marla L. Clayman ${ }^{4}$
}

Published online: 9 March 2016

(C) Springer Science+Business Media New York 2016

Erratum to: J Cancer Surviv

DOI 10.1007/s11764-016-0524-9

Unfortunately, there is an error occurred in the first name of the first author Andrea M. Russell in the online published version. The author name has been corrected with this erratum.

The online version of the original article can be found at http://dx.doi.org/ $10.1007 /$ s11764-016-0524-9.

Marla L. Clayman

mclayman@air.org

1 Division of General Internal Medicine, Feinberg School of Medicine, Northwestern University, Evanston, IL, USA

2 Department of Communication Studies, Northwestern University, Evanston, IL, USA

3 Biomedical and Health Information Sciences, College of Applied Health Sciences, University of Illinois at Chicago, Chicago, IL, USA

4 American Institutes for Research, Chicago, IL, USA 